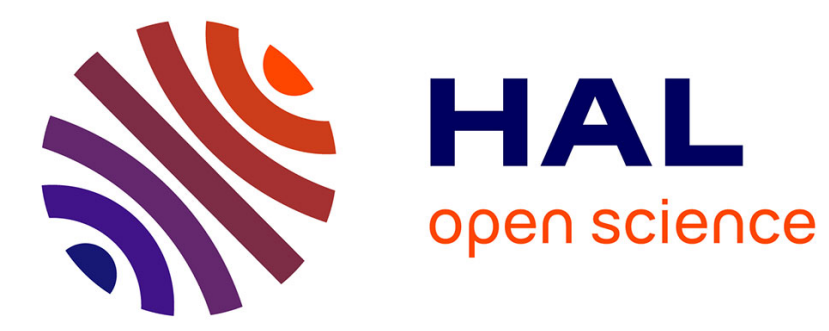

\title{
Optical Power Control in GMPLS Control Plane
}

Mohamad Kanj, Esther Le Rouzic, Julien Meuric, Bernard Cousin, Djamel

Amar

\section{To cite this version:}

Mohamad Kanj, Esther Le Rouzic, Julien Meuric, Bernard Cousin, Djamel Amar. Optical Power Control in GMPLS Control Plane. Journal of Optical Communications and Networking, 2016, 8 (8), pp.553 - 568. 10.1364/JOCN.8.000553 . hal-01427052

\section{HAL Id: hal-01427052 https://hal.science/hal-01427052}

Submitted on 5 Jan 2017

HAL is a multi-disciplinary open access archive for the deposit and dissemination of scientific research documents, whether they are published or not. The documents may come from teaching and research institutions in France or abroad, or from public or private research centers.
L'archive ouverte pluridisciplinaire HAL, est destinée au dépôt et à la diffusion de documents scientifiques de niveau recherche, publiés ou non, émanant des établissements d'enseignement et de recherche français ou étrangers, des laboratoires publics ou privés. 


\title{
Optical Power Control in GMPLS Control Plane
}

\author{
Mohamad Kanj, Esther Le Rouzic, Julien Meuric, Bernard Cousin, Member, IEEE, and Djamel Amar
}

\begin{abstract}
The exponential traffic growth in optical networks has triggered the evolution from Fixed-Grid to Flex-Grid technology. This evolution allows better spectral efficiency and spectrum usage over current optical networks in order to facilitate huge dynamic traffic demands. The promise of Flex-Grid technology in terms of increasing the number of optical channels established over optical links may however not be sustainable because of the associated increase in optical amplification power. In this work, we detail a power control process that takes advantage of link optical power and channel optical signal to noise ratio (OSNR) margins to allow network operators to support this optical power increase while maintaining the use of legacy optical amplifiers. New GMPLS protocol extensions are proposed to integrate the optical power control process in the control plane. The performance of the process is evaluated in terms of the blocking ratio and network throughput over Fixed-Grid and Flex-Grid networks. Results show that controlling optical power benefits from the Flex-Grid technology in terms of spectrum and capacity gain and reduces optical connection blocking.
\end{abstract}

Index Terms-GMPLS, OSPF-TE, RSVP-TE, Flex-Grid, Optical power control, Optical link design.

\section{INTRODUCTION}

I NTERNET services (e.g., video conferencing, cloud services, and video streaming) and consequently traffic demands are increasing continually, leading to huge traffic growth in the core optical network. There is a need for network operators to increase their optical network capacity to follow this traffic growth. Since the deployment of new optical fibers is still very expensive, network operators are pushing to exploit the totality of their network capacity by optimizing their optical resources, and thus postponing the deployment of new optical infrastructures. This exploitation requires new technologies and flexible equipment that are able to handle different types of optical channels, from small to extremely high data rates [1].

Fixed-Grid technology is no longer qualified to handle the increasing data rates of optical channels. At the same time, the $50 \mathrm{GHz}$ ITU-T grid, due to its fixed-spectrum spacing, leads to spectrum inefficient usage when the spectral bandwidth of the optical channels is smaller than the size of the allocated $50 \mathrm{GHz}$ slot [2]. The ITU-T recommendation G.694.1 [3] for a Flex-Grid optical network has defined a new flexible spectral grid standard for wavelength division multiplexing (WDM) applications. This flexible spectral grid has a smaller slot granularity of $12.5 \mathrm{GHz}$, with nominal central frequency

M. Kanj is with $\mathrm{b}<>$ com, 1219 avenue Champs Blancs, 35510 CessonSevigne, France e-mail: Mohamad.KANJ@b-com.com.

B. Cousin is with IRISA Labs, University of Rennes 1, 35000 Rennes, France.

E. Le Rouzic and J. Meuric and D. Amar are with Orange Labs Lannion, 22300 Lannion, France.

Manuscript received April 19, 2005; revised January 11, 2007. on a grid of $6.25 \mathrm{GHz}$ spacing compared to the current 50 $\mathrm{GHz}$ Fixed-Grid.

This recommendation has transformed the Flex-Grid into a promising technology that is capable of following traffic growth and various traffic demands. Flex-Grid efficiently uses available spectrum resources, especially when associated with novel coherent transmission technologies and advanced modulation formats. In addition, since Flex-Grid technology allows the reduction of channel spacing, it offers the possibility to create new optical channels over the saved spectrum. However, increasing the number of optical channels increases the optical power injected in optical links, which may not be acceptable in some of the already deployed amplifiers.

Indeed, this increase in optical power, when switching from Fixed-Grid to Flex-Grid technology has an effect on the legacy optical amplifiers. It could cause amplifier saturation and dramatic performance degradation for the already established channels (probably leading to transmission failure). Therefore, there is a need to replace the existing amplifiers by new ones with bigger output powers. However, the deployment of new flexible transponders, powerful optical amplifiers and new flexgrid wavelength selective switches, in addition to the operational cost, makes the Flex-Grid technology very expensive for network operators in spite of its capacity increase promises.

In this respect, we demonstrated in [4] that if we control the power of the optical channels, it is possible to keep the existing amplifiers when migrating to Flex-grid technology. Moreover, this power control allows $10 \%$ of cost reduction with respect to conventional Fixed-Grid, without mentioning the saved cost through avoiding the purchase and the deployment of new amplifiers and the service interruption of the optical links.

\section{A. Related Works}

In the literature, several studies have focused on developing accurate physical impairment estimators over uncompensated links [5][6][7][8]. They have demonstrated the existence of an optimal optical channel power that leads to minimum impairment generation and thus achieves better transmission performance (maximum reach).

In general, during the offline system design, every physical link between two adjacent optical nodes is designed to support a maximum capacity while maximizing the optical reach for all channels, through the use of this optimal power per channel (usually different optimal power per link since it depends on the length and the attenuation of the optical spans constituting the link). However, the resources provisioning for the worst case (i.e., full capacity, and maximum transmission reach) consequently leads to power resource over-dimensioning with considerable power margins on some links, due to the nonuniform distribution of traffic demands and their required reaches. 
In this respect, many recent studies focused on improving link performances (i.e., minimizing the nonlinear interference effect) and thus increasing network throughput by adjusting channel launch power and optimizing spectral resources using several modulation formats [9][10][11][12]. Others in [13][14], focused on adapting launch powers depending on required data rates and the reach in order to reduce overall network cost and saving the number of signal regeneration. However, the practical feasibility of such adaptation was not proposed yet, more precisely from a control plane point of view.

All these studies, in addition to the ones dealing with the control plane of Flex-Grid networks [15][16], have only considered the spectral resources as a limitation without taking into account the power resource limits of optical links (which depend on the deployed amplifiers). They proposed a control plane algorithm that takes only into account, the transparent spectrum assignment and the physical feasibility of the optical channels. However, despite the demonstrated benefits from controlling the power of optical channels, there is no routing algorithm suggested until know with suitable control plane architecture, in order to allow the practical implementation of such channel power adaptation.

\section{B. Contributions}

For all these mentioned reasons, in this work, and unlike the current paradigm, we take into account optical power resource limits in addition to the spectral ones. Moreover, we propose the practical feasibility of such solutions through a power and impairment aware routing algorithm, in addition to protocol extensions in order to make the relationship between planning and control plane. The proposed control process adapts the power of optical channels to their minimum required performances (adaptation to the real physical reach). This adaptation enables optical power margins to be used for overcoming the power limitations of amplifiers when increasing the number of channels over network links.

Therefore, a new path computation algorithm is developed for a distributed generalized multi-protocol label switching (GMPLS)-based control plane. Original protocol extensions are proposed to resource reservation protocol-traffic engineering (RSVP-TE) and open shortest path first-traffic engineering (OSPF-TE) to collect new physical parameters and to enable the use of the power control process. The performance of the novel scheme is demonstrated with simulations, by evaluating the cumulative blocking ratio (CBR) and network throughput.

It noteworthy that this routing algorithm is completely compatible with the Software Defined Network (SDN) paradigm, since it could be executed by an SDN controller if the same collected information through OSPF-TE was stored in the controller database.

This work extends a previous study presented in [17] by introducing protocol extensions and describing signaling message details and the mechanisms used to integrate these extensions in a distributed GMPLS control plane. Moreover, we produce additional performance evaluations (for instance, the effect of the number of shortest paths) and enrich our previous work with a deeper analysis of the blocking reasons for six simulated scenarios.

This paper is organized as follows. Section II presents an overview of optical link design issues, recalls our link design method, and introduces the link power margin. Section III presents our power control process. Section IV presents our new path computation algorithm. Section V presents our OSPF-TE and RSVP-TE protocol extensions to implement the power control process in a GMPLS control plane. It also presents the signaling mechanism through a channel connection establishment example. Section VI presents simulated scenarios and results, in addition to blocking reasons analysis. The conclusion and future works are presented in Section VII.

\section{OPTICAL LINK DESIGN AND POWER LIMITATIONS}

We consider a set of successive optical spans constituting an optical link $l$ between two optical nodes (e.g., reconfigurable optical add-drop multiplexers; ROADMs) as shown in Fig.1. The optical link design consists of choosing the set of optical amplifiers that can compensate for span losses and simultaneously support the aggregated optical power of all the channels planned for that link, while seeking maximum optical performance. The link design has the objective of maximizing OSNR, through minimizing linear and non-linear effects. The complexity of the process arises in particular because of the contradictory objectives of amplifiers; they must compensate for link span losses, satisfy the aggregate optical power for all optical channels sharing the fiber, and simultaneously minimize the amount of generated noise.

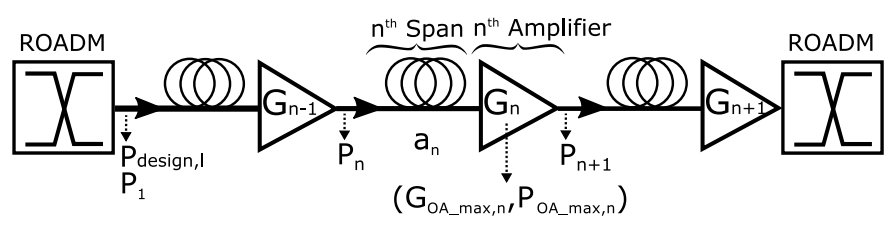

Fig. 1. Simplified representation of an amplified link (succession of a fiber span and optical amplifier) between two ROADMs.

In general, optical links in Fixed-Grid WDM networks are designed to support a given number of channels $N_{\text {channel_max }}$ (e.g. 80 channels with $50 \mathrm{GHz}$ of spectral occupation for 4 THz of optical bandwidth). This $N_{\text {channel_max }}$ is equivalent to an aggregated optical power, which in turns depends on the calculated per channel optimal power during the design step. Usually, each link $l$ in the network has the same $N_{\text {channel_max }}$, except when network operator have a particular need over certain links. However, to ease our study, without any loss of generality, we assume that these numbers are identical all over the network. It is important to note that, we do not have necessarily for every link $l$ the same channel optimal power, since it depends on the physical characteristics of each link (i.e. spans attenuation, amplifiers configuration).

The use of Flex-Grid technology over these optical infrastructures may increase the number of channels in some links and thus their optical power levels (e.g. Up to 106 channels with $37.5 \mathrm{GHz}$ of spectral occupation in $4 \mathrm{THz}$ of bandwidth 
and up to 128 channels in $4.8 \mathrm{THz}$ of bandwidth). In fact, if the number of channels (i.e. the aggregated optical power for the $N_{\text {channel_max }}$ channels) is not controlled and limited, there may be some risks of power saturation in the amplifiers that are already close to their maximum output power (i.e., power saturation limit) leading to strong performance degradation on these links.

Inversely, if the number of channels is limited to $N_{\text {channel_max }}$, the spectrum gain enabled by Flex-Grid technology cannot be exploited. However, replacing optical amplifiers with new ones having bigger output power is a potential solution, but costly since it requires full redesign of the links and possible purchase of new amplifiers as well as the interruption of the link to set up the new amplifiers configuration.

In this paper, we propose making the information of optical power available to the control plane to benefit from the Flex-Grid spectrum gain promises, while keeping the inplace amplifiers. However, this requires fine knowledge of the maximum power allowed in each link, which in turn requires understanding the link design step and the limitations of optical links.

\section{A. Design Method}

In order to evaluate our power control process, we must precisely model the link design step. The design of optical links (i.e. selection/configuration of optical amplifiers) is an important phase to determine optical resources limits over every optical link. To this end, we have developed a link design method, which we presented in [4][17][18], taking advantage of the optimization strategy presented in [19]. Surely, any other design method could be used instead to determine these power resources. In this case, the estimator of the physical feasibility used in the control plane will be different since it depends on the design method.

The LOGON strategy proposed in [19] consists of performing a local optimization of the OSNR and non-linear impairments at span level, leading to a global OSNR optimization over all the links of the network. It proposes applying an optimal power spectral density (optimal DSP) on every channel at the input of every span to guarantee maximum transmission performance over the channels. This power is calculated using span and amplifier characteristics by applying equation 6 in [19].

Our link design method developed in [17][4], is based on an analytical formula that calculates amplifier gains while respecting optimal powers to be set at the input of optical spans, thus leading to link OSNR optimization. After this link design phase (or any other design phase), every link has its own set of amplifier types with various power and gain settings, which subsequently determines the power resource limits and the quality parameter of the link (i.e., OSNR).

It is important to note that amplifiers are used in a fixed gain mode, which means that once the design phase is finished, amplifier gain settings are never changed. Furthermore, to efficiently manage optical power resources, many essential parameters should be available to the control plane of the network. Therefore, in order to understand these parameters, we study the existing power resources available over the optical links after the application of our design method.

\section{B. Link Power Margin}

Let $N_{\text {channel_max }}$ be the maximum number of channels per link. Let $P_{\text {design,l }}$ (by definition equal to $P_{1}$ as shown in Fig. 1) be the input optical power designed for the link $l$ having $N_{\text {channel_max }}$. The difference of characteristics between all spans in terms of the losses, non-linearity coefficient, and length, leads to the use of various types of amplifiers having different characteristics in terms of maximum gain $\left(G_{O A \_\max }\right)$, maximum power $\left(P_{O A \_\max }\right)$, and noise figure $(N F)$. This difference results in different $P_{\text {design,l }}$ and thus

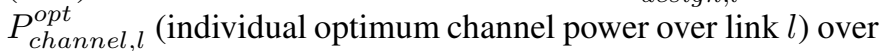
every link $l$ and in different span optimum input powers (i.e., amplifier output powers). This power variation is given by:

$$
G_{n}=a_{n} \frac{P_{n+1}}{P_{n}}
$$

where $G_{n}$ is the gain of the $n^{t h}$ amplifier, $a_{n}$ is the attenuation of the $n^{t h}$ span, $P_{n}$ is the power at the input of the $n^{t h}$ span, and $P_{n+1}$ is the power at the input of the $n+1$ span (output power of the $n^{t h}$ amplifier) as shown in Fig. 1. Therefore, it may remain a power margin $\left(P_{O A \_} \operatorname{margin}, n\right)$ over the $n^{t h}$ amplifier such that:

$$
P_{O A \_m a r g i n, n}=P_{O A \_m a x, n}-P_{n+1}
$$

where $P_{O A \_\max , n}$ is the maximum power of the $n^{t h}$ amplifier. Fig. 2 shows an example of power levels $\left(P_{O A \_m a x}, P_{O A \_m a r g i n}\right)$ over link $l$ amplifiers, where different $P_{O A} \_$margin values exist in the different amplifiers.

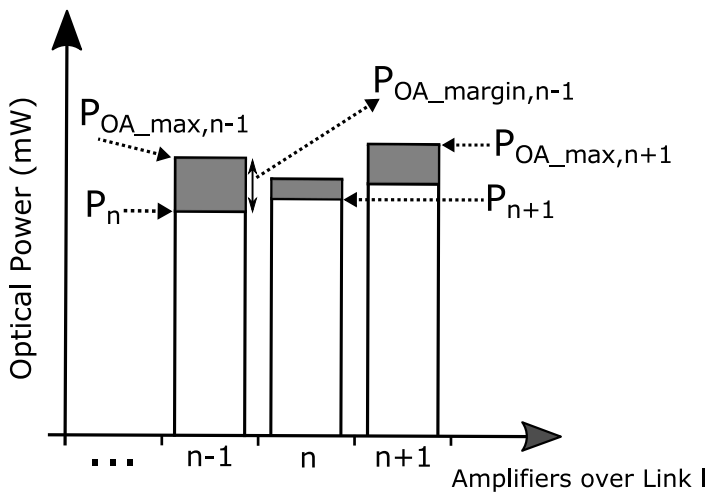

Fig. 2. A simplified representation of optical power at amplifier level.

We define as link power margin $P_{\text {margin, }}$, the minimum power margin (minimum $P_{O A \_m a r g i n}$ ) that exists over the amplifiers of the link $l$ :

$$
P_{\text {margin }, l}=\min _{n}\left\{P_{O A \_m a r g i n, n}\right\} \quad \forall n \in \aleph_{l}
$$

Where $\aleph_{l}$ is the set of amplifiers of the link $l$. Therefore, the maximum optical power that can be applied at the input of link $l$ without saturating any amplifier is

$$
P_{\text {max }, l}=P_{\text {design }, l}+P_{\text {margin }, l}
$$


In case there is no $P_{O A \_m a r g i n, n}$ in one of the amplifiers of the link $l$, the $P_{\text {margin, } l}$ is then equal to zero (in $\mathrm{mW}$ ), and no additional power can be used over that link. We define $P_{l}(t)$ as

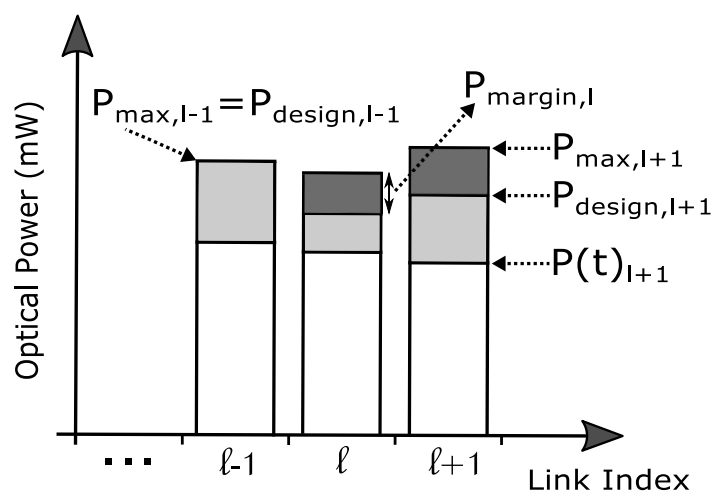

Fig. 3. Simplified representation of power levels over optical links.

the current power at a moment $t$ over the link $l$. It is a function of time $t$ since it depends on the number and on the power of the established channels until time $t$. Fig. 3 illustrates the different power levels that can exist over the optical links of a network, where $l$ is the link index. For every link a $P_{\max , l}$ power is supported, where $P_{\max , l}$ is greater than $P_{\text {design,l }}$ of the link (by construction). It is exactly equal to $P_{\text {design,l }}$ when no power margin exists. As shown in Fig. 3, the link $l$ and $l+1$ have strictly positive margins; therefore, additional optical resources may be used over these links. Inversely, the link $l-1$ does not have power margin; therefore, no additional power can be used over this link. Our utilization of power margin complements recent works on design margins and system margins, as in [20][21][22]. In these works, the power control aspect was neglected. Here, we specifically focus on the control of the optical power.

\section{OPTICAL POWER CONTROL}

Optical networks are made of optical nodes (ROADMs) interconnected with optical links. In order to achieve maximum network performance, every optical link between two ROADMs is usually designed to support optimum performance independently from other links. With this design method, every link has its own set of optimum span powers and amplifier settings. The maximum performance is ensured by setting the optimum power for any new optical channel (i.e., $P_{\text {channel,l }}^{\text {opt }}$ [19]). This kind of policy does not consider that channels may require variable reaches; thus, some channels may not always need the maximum performance (e.g., the power of the channel with the shortest path does not need to be set to its optimal value to reach the destination). As a result, some transmission margins are wasted.

Channel performance and its optical power are tightly linked. Reducing the optical power from its optimum value to a lower value reduces the performance and thus adapts the channel to the required reach. This appears as an interesting method to save some optical power in a Flex-Grid network and to avoid wasting transmission margin. More precisely, we expect that this power adaptation will allow the use of the transmission margin to increase link capacity in terms of channel numbers.

To perform the power control, we now propose exploiting the performance estimator of equation 5 of the LOGON strategy in [19]. This equation estimates the OSNR (including non-linear effects in the form of non-linear interference) of a lightpath $p$ at the receiver side. The $O S N R_{e s t, p}$ value of the lightpath $p$, which is made of $m$ successive links, is the inverse of the sum of the inverse $O S N R$ of each link [19]. If the estimated OSNR $\left(O S N R_{e s t, p}\right)$ is bigger than that required $\left(O S N R_{r e q, p}\right)$, then the channel power can be adapted. We define the $O S N R_{\text {margin }, p}$ as the difference between the estimated and required OSNR:

$$
O S N R_{\text {margin }, p}[d B]=O S N R_{e s t, p}[d B]-O S N R_{r e q, p}[d B]
$$

It is noteworthy that LOGON assumes the worst case in terms of nonlinear effects (i.e., OSNR overestimation assuming full spectrum load), which means that the establishment of any new channel will not require the recalculation (i.e., re-estimation) of the OSNR for the already established ones, since its effect is already considered.

The OSNR of an optical channel varies in function of its optical power at the transmitter side: $O S N R=f\left(P_{\text {channel }}\right)$. The function $f$ is monotonically increasing on the interval $\left[0, P_{\text {channel,p }}^{\text {opt }}\right]$ [23], where $P_{\text {channel, } p}^{\text {opt }}$ is the channel transmit power for optimal reception of light at the destination of the path $p$ (i.e., the channel optimum power on the first link constituting the path $p$ ).

In order to translate power reduction into OSNR reduction, we have considered that every $1 \mathrm{dBm}$ of optical power reduction corresponds to $1 \mathrm{~dB}$ of OSNR reduction. This is an overestimation to ensure a working channel. Indeed, $1 \mathrm{dBm}$ of power reduction leads to less than $1 \mathrm{~dB}$ OSNR reduction as explained in [23]. Therefore, we can consider that the OSNR margin in $\mathrm{dB}$ corresponds to the amount of power that can be saved for the related optical channel. Moreover, since optical amplifiers have fixed gains (adjusted according to the method explained earlier in II-A), this OSNR reduction is obtained by tuning the power at the transmitter side. An $x \mathrm{dBm}$ of optical power attenuation at the transmitter side corresponds exactly to $x \mathrm{dBm}$ of power attenuation at the receiver side, when passing though the set of spans and amplifiers constituting the optical link. With this method, we obtain the adapted channel power:

$$
P_{\text {channel }, p}^{\text {adapted }}=P_{\text {channel }, p}^{\text {opt }} \times \frac{O S N R_{r e q, p}}{O S N R_{e s t, p}}
$$

We define the channel power adaptation value as $C_{\text {adaptation: }}$ :

$$
C_{\text {adaptation }, p}[d B]=\beta \times O S N R_{\text {margin }, p}[d B]
$$

where $\beta \in[0,1]$ and $C_{\text {adaptation, } p}$ represent the quantity of OSNR degradation to apply over the lightpath $p$. In our work, we use $\beta=1$. However, $\beta$ can be used to introduce flexibility to the channel power adaptation process. It offers the possibility for the control plane to efficiently manage its transmission power margins.

The estimation of the power that can be saved is a simple calculation that can be easily integrated into a control plane. 


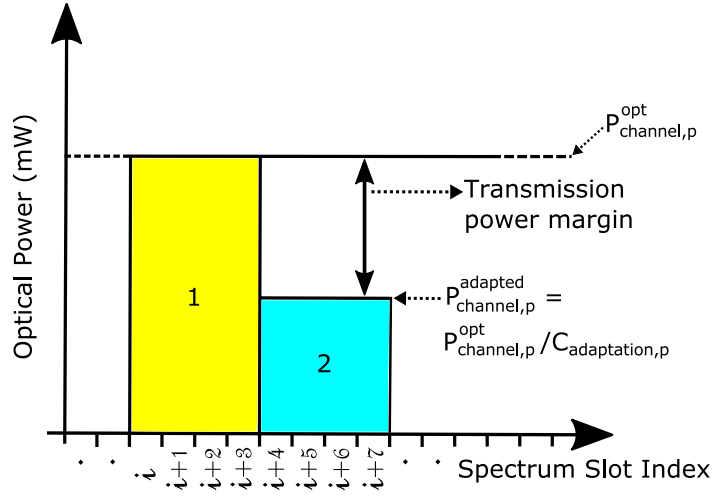

Fig. 4. Optical channels with and without power adaptation.

Other methods relying on more complex computation or monitoring mechanisms can be used to estimate the OSNR. However, this is out of the scope of this paper.

To illustrate the power control process, we assume that two optical channels having the same spectral occupation of 50 $\mathrm{GHz}$ (i.e., four slots of $12.5 \mathrm{GHz}$ ), are established over the same path $p$. The first channel is established without power adaptation and the second with power adaptation. Fig. 4 shows an example of the optical power level at the transmitter side, for the two established channels. In this example, the first channel (in yellow) uses its optimal power $P_{\text {channel, } p \text {. The }}^{\text {opt }}$ second channel (in blue) is adapted to minimum acceptable performance $O S N R_{\text {req }}$, and its power value is calculated using (6)

\section{ROUTING ALGORITHM}

To find an optical path between a node pairs, we propose a new path computation algorithm that considers spectral and power resources and performs a power adaptation process. Fig. 5 shows the algorithm, which is executed at the ingress node during path calculation.

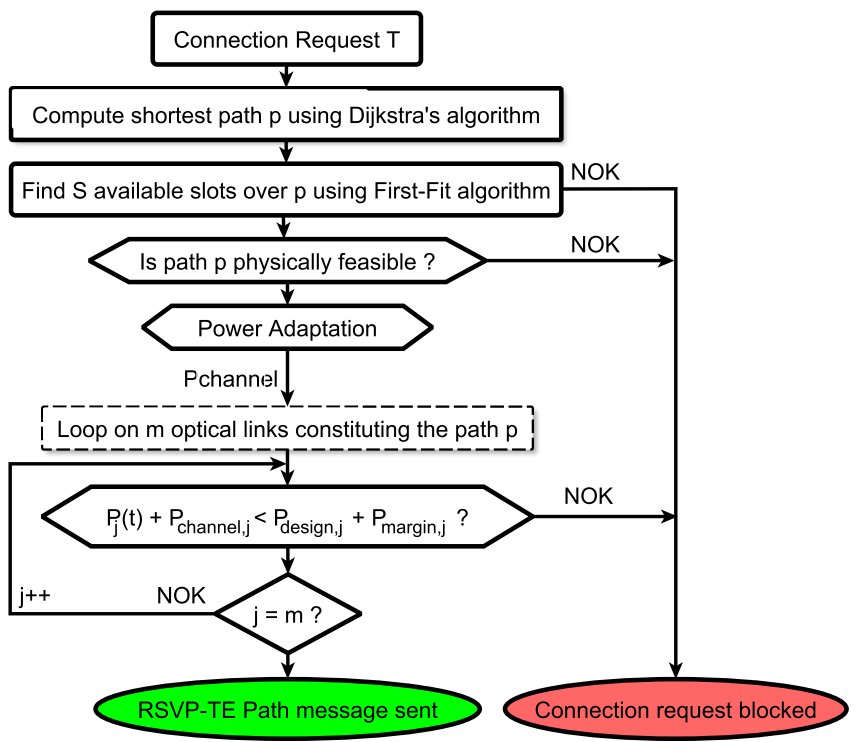

Fig. 5. Path Computation Algorithm.
For every optical connection request (i.e., lightpath establishment request) of $T$ Gbit/s rate between a pair of source and destination nodes, it calculates the shortest path using Dijkstra's algorithm. Then, it tries to find a group of $S$ continuous and contiguous available slots of $12.5 \mathrm{GHz}$ that satisfies the request $T$ using the First-Fit algorithm. The $S$ slots are calculated with respect to minimum spectrum occupation, supposing the same modulation format and baud rate for each request in this study. The request is blocked when no available slots are found to satisfy the connection request. However, once this set of available and successive optical slots over a path $p$ is found, three other tests are performed: the physical feasibility, power adaptation (PA), and power verification (PV) tests. The physical feasibility test checks whether $O S N R_{e s t, p}>O S N R_{r e q, p}$. If the path is physically feasible, then $O S N R_{\text {margin, } p}$ is computed. If $O S N R_{\text {margin, } p}>0$, then the channel optical power is adapted to minimum acceptable performance $O S N R_{r e q, p}$. Therefore, the channel OSNR degradation value

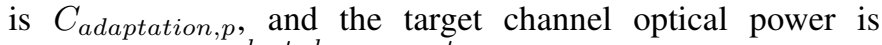
expressed as $P_{\text {channel }, p}^{\text {adapted }}=P_{\text {channel }, p}^{\text {opt }} / C_{\text {adaptation }, p}$.

Regardless of the adapted channel power value, a last power verification test is performed to ensure that this channel, if added, will not cause any saturation problems over the $m$ links constituting the optical path $p$. This test consists of comparing for every link of the optical path $p$, the link aggregate power $P_{l}(t)$ after adding the power of the new channel (either $P_{\text {channel, },}^{\text {opt }}$ if no power adaptation is performed or $P_{\text {channel, } l}^{\text {adapted }}$ if power adaptation is performed) with the maximum allowed power $\left(P_{\max , l}\right)$. It is important to note that these power parameters $\left(P_{\text {design, } l}, P_{\text {margin, },}, P_{\text {channel, },}\right.$, and $\left.P_{l}(t)\right)$ are made available at each node thanks to the extension that we propose for the OSPF-TE link state distribution process. Once these tests are done at the ingress node, the signaling is triggered on the chosen path (i.e., an extended RSVP-TE Path message containing the power adaptation information is sent downstream in order to set up the optical channel. If any of these tests fail, the connection request is rejected.

Lastly, at each hop, during the signaling process, the aggregate power using the recommended channel power setting is checked in order to verify that it does not exceed the $P_{\max , l}$ of each crossed link. Indeed, if the requests are very frequent, some signaling process may simultaneously compete for the same optical resources in terms of optical power (race condition) and the signaling should avoid any overprovisioning due to the not-yet-updated link database.

\section{GMPLS PROTOCOL EXTENSIONS}

The GMPLS is a network protocol suite for setting up connectivity services upon different switching type equipment [24]. GMPLS is used here because it is one of the most deployed control plane and since it is widely used to manage the optical networks technologies. Moreover, it is a welldefined and stable protocol suite involving signaling, routing and link management protocols to automatically provision end-to-end traffic-engineered connections.

In this section, we mainly focus on the OSPF-TE topology distribution and RSVP-TE signaling protocols, which are the 
main required bricks of our optical power control process. Despite recent efforts of IETF to enrich GMPLS control plane with extensions for Flex-Grid networks[25][26] and with physical layer awareness [27], neither the parameters that we need for the optical power control process, nor the process itself are defined. Therefore, in this work, we propose adding several extensions for OSPF-TE and RSVP-TE protocols. Subsequently, we provide the detailed description of these extensions and present the routing and signaling mechanisms used to exploit them.

\section{A. OSPF-TE Extensions}

At the end of the design phase, every optical link has its own set of characteristics, which are: $P_{\text {channel,l }}^{\text {opt }}, P_{\text {design, },}$, $P_{\text {margin, } l}$, and link $O S N R_{l}$. We assume that, in the initialization phase of the network, these physical parameters are recorded for each link in the neighboring nodes upon link commissioning. Then, they are collected via OSPF-TE flooding control messages and placed in a local database in each node.

As described in IV, in order to realize the power verification test, another parameter is needed, which is $P_{l}(t)$. It is added in the local database, with a value that corresponds to 0 $m W$ during the initialization phase of the network because no channel has been established yet.

In this respect, we propose five new sub-TLVs to OSPF-TE link TLV:

- $P_{\text {channel, }}(\mathrm{dBm})$ : the input optimum power for the reference channel spacing $(50 \mathrm{GHz})$ over the link $l$. It is used when no power adaptation is applied.

- $P_{\text {design,l }}(\mathrm{dBm})$ : the total aggregated input power designed for the $N_{\text {channel_max,l }}$ of the link $l$. This parameter is required by the control plane to determine the aggregated optical power allowed over the link $l$.

- $P_{\text {margin }, l}(\mathrm{dBm})$ : the link optical power margin. This parameter represents the remaining power margin over the link $l$.

- OSNR $(\mathrm{dB})$ : the OSNR of the link $l$ as defined in equation 5 of [19]. This parameter is needed to estimate path feasibility during the path computation process. Note that this parameter is slightly different from the one proposed in [28][29], since it includes a nonlinear effects contribution.

- $P_{l}(t)(\mathrm{dBm})$ : the link power of link $l$ at time $t$. This parameter is used by the power verification test.

We propose to include the first four sub-TLVs into the Opaque link state advertisement (LSA) type 8 ("OSPFv2 Extended Link Opaque LSA"). The $P_{l}(t)$ sub-TLV is included as part of the Opaque LSA type 1 ("Traffic Engineering LSA"). We propose to encode every one of these five sub-TLVs over 8 bytes, where the first 2 bytes are used to indicate the type of sub-TLV and the second 2 bytes are used to indicate the length of the sub-TLV (which is equal to 4 here). The last 4 bytes are used to encode the value field of the sub-TLV with respect to the 32-bit IEEE floating point format. In addition to the proposed sub-TLVs, we also rely on an additional subTLV to take into account the spectrum slot availability. Many coding formats were proposed for the slot availability sub-TLV in the IETF draft [25][30]. We adopted the bit map format in this work.

During the creation of the local databases, we separated the record for the static ( $P_{\text {channel,l }}^{\text {opt }}, P_{\text {design,l }}, P_{\text {margin, },}$, $O S N R_{l}$.) and dynamic $\left(P_{l}(t)\right.$, spectrum slot availability bit map) parameters [31]. The proposed static parameters are never changed during network operation except in the case where link design or equipment (amplifier or link) was changed (e.g., in the case of fiber repair). The dynamic parameter values change every time an optical channel is established or released. This separation allows the reduction of the amount of flooded information through OSPF-TE protocol.

It is noteworthy that additional parameters may be added to enrich the physical layer awareness, such as chromatic dispersion (CD) or polarization mode dispersion (PMD) of the optical links as proposed in [32]. However, these parameters are out of scope of our study, since they have no direct relationship with our power control process. They may be included to improve the exactness of the physical feasibility evaluation of the lightpath.

\section{B. RSVP-TE Extensions}

In the GMPLS protocol suite, the RSVP-TE protocol is used as the signaling process between optical nodes of the calculated path to establish the requested connection. We adopted the already proposed RSVP-TE extensions by the IETF in [26] for the Flex-Grid optical networks.

These extensions are used to represent slot width (i.e. bandwidth occupation of the channel) and the frequency slot information. The slot width extension is used to represent how much spectrum resource is requested for a Label Switched Path (LSP). The frequency extension is used to identifies the location of the channel in the spectrum of the optical link.

After the path computation procedure, the ingress node sends an RSVP-TE Path message to the next node of the calculated path. This Path message contains information on connection to setup: the central frequency, the channel width (i.e., number of slots) and $C_{\text {adaptation, } p}$ value. When a node receives a Path message (or Resv message), two tests are performed over its outgoing links: the slots availability verification and the optical power verification.

The slots availability verification consists of verifying that the requested slots are not occupied by any other optical channel. The power verification tests whether the requested power does not exceed the link maximum power. Therefore, it uses the $C_{\text {adaptation,p }}$ value conveyed through Path and Resv messages in combination with $P_{\text {channel, }}^{\text {opt }}$ value recorded in its local database to compute the requested power of the connection. Then, it determines whether if the power respects the following constraint:

$$
P_{l}(t)+\left(P_{\text {channel }, l}^{\text {opt }} / C_{\text {adaptation }, p}\right) \leq P_{\text {design }, l}+P_{\text {margin }, l}
$$

The $C_{\text {adaptation }}$ parameter is conveyed through Path and Resv messages because it is only known by the ingress node (during path computation) and is not distributed by OSPF-TE. To this end, we propose to create new 8-byte sub-TLVs (two 
bytes for type, two bytes for length, and four bytes to encode the value) in each of the $S E N D E R \_T S P E C$ (Class number 12) and FLOW SPEC (Class number 9) objects of the Path and Resv messages, respectively. These sub-TLVs contain the

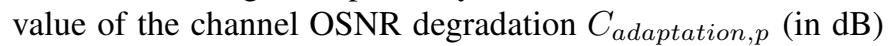
for a path $p$, which is encoded respecting the 32-bit IEEE floating point format.

It is important to note that during the RSVPTE signaling process, each node of the optical path stores channel information contained in the Path and Resv messages in a local database (referred to as "PathStateBlock/ReservationStateBlock" in the standard). Therefore, when an optical channel is removed, optical nodes use the stored information to release the optical resources of the channel (i.e., occupied slots). Simultaneously, it also update the values of $P_{l}(t)$ for the concerned links, using $C_{\text {adaptation, } p}$ parameter combined with $P_{\text {channel, }}^{\text {opt }}$ to calculate the value of the optical power to be subtracted from $P_{l}(t)$.

In the same context, the integration of the power verification test requires the addition of a new type of error in case the test fails. Therefore, we propose to define a new error code for the ERROR_SPEC (Class number 6) object of the RSVPTE PathErr message [33]. This allows the identification of the error by the ingress node in order to indicate that the link power resource is fully used.

\section{Connection Establishment Example}

To explain the control mechanism used in our work, we consider here, as an example, an optical network with six optical nodes (i.e., ROADMs). Fig. 6 shows the six interconnected nodes (A, B, C, D, E, and F).

We assume that network optical links are already designed and that the nodes database is filled with essential information ( $P_{\text {channel, }, l}^{\text {opt }}, P_{\text {design, },}, P_{\text {margin }, l}, O S N R_{l}$, and $P_{l}(t)$ ). Moreover, we suppose, in this example, that a connection request between ROADMs $\mathrm{A}$ and $\mathrm{C}$ is sent from the network operator to Node A. Fig. 7 shows the signaling mechanism and the RSVP-TE message flow triggered to establish the optical channel.

Upon receipt of the connection request by Node $\mathrm{A}$, the path computation algorithm is triggered. We assume that, after performing the algorithm, the selected path $p$ is A-BC (shortest path), and $S$ free available slots are found. We suppose also that the $O S N R_{e s t, A B C}$ of the path is bigger than $O S N R_{r e q, A B C}$. Therefore, the optical channel for the path $p$ is power adaptable and a $C_{\text {adaptation, } A B C}$ parameter is computed.

Before triggering the RSVP-TE signaling process, Node A performs the slot and power verification tests over its outgoing link (i.e., AB). These tests are executed to ensure that optical spectrum resources are still available and no power saturation will occur after adding the new optical channel over link $\mathrm{AB}\left(P_{A B}(t)+\left(P_{\text {channel, } A B}^{\text {opt }} / C_{\text {adaptation, } A B C}\right) \leq\right.$ $\left.P_{\text {design, } A B}+P_{\text {margin, } A B}\right)$. Once verification is done, Node $\mathrm{A}$ sends an RSVP-TE Path message to Node B with the same information on the selected path $p$ (A-B-C), the $S$ slots, and

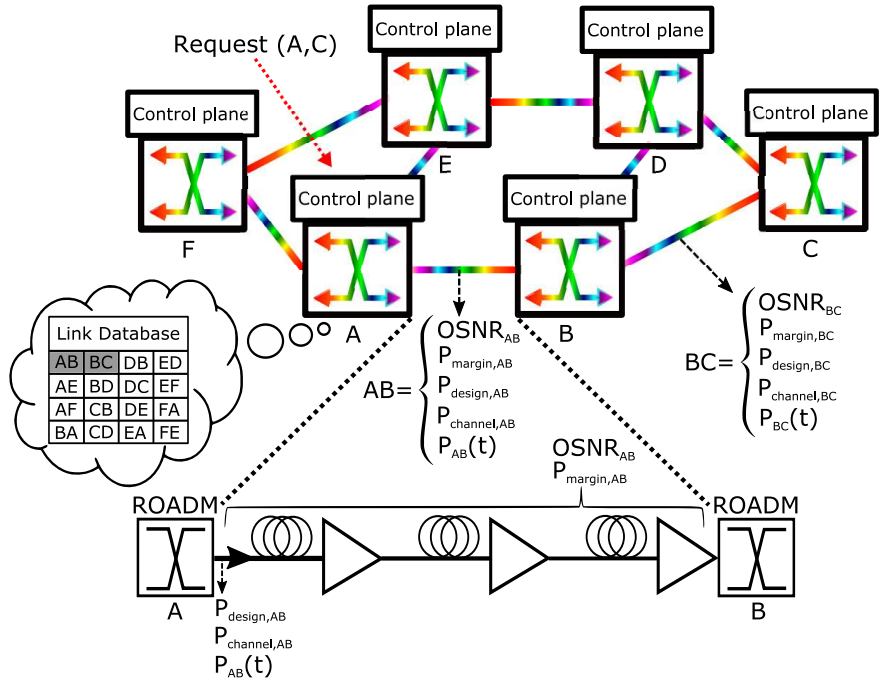

Fig. 6. Network example.

the $C_{\text {adaption, } A B C}$ value. Upon reception of the Path message by Node B, the same tests are performed over its outgoing link, BC (it checks that $S$ are still available over the link $\mathrm{BC}$, and that $P_{B C}(t)+\left(P_{\text {channel, } B C}^{\text {opt }}-C_{\text {adaptation, } A B C}\right) \leq$ $\left.P_{\text {design, } B C}+P_{\text {margin, } B C}\right)$. Then, it sends a Path message to Node $\mathrm{C}$, once the verification is done.

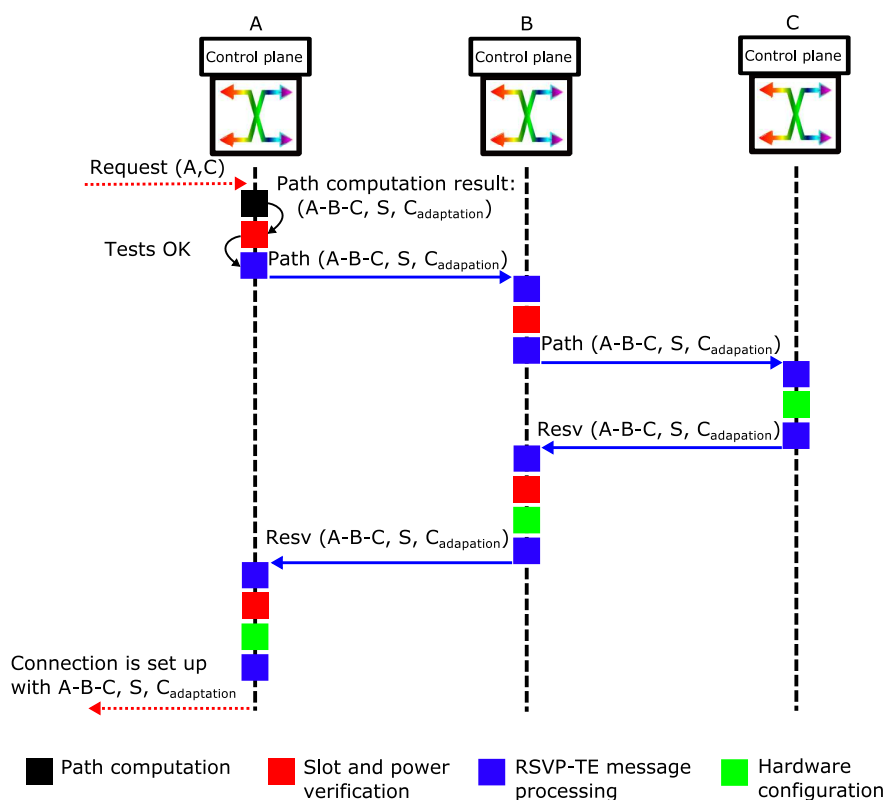

Fig. 7. Flow diagram in $\mathrm{A}, \mathrm{B}$, and $\mathrm{C}$ controller during the connection provisioning process.

Once the Path message arrives to the egress Node C, a hardware configuration is performed for its Drop port (in order to receive the optical channel). Moreover, the spectrum bit map and the power value of the link $\mathrm{BC}$ are updated $\left(P_{B C}(t)=P_{B C}(t)+P_{\text {channel, } B C}^{\text {opt }} / C_{\text {adaptation, } A B C}\right)$ in its local database. Then a Resv message is sent to Node B. On receipt of the Resv message by Node B, the slot availability and power verification tests are performed again over link BC. Then, a hardware configuration is made to ensure the switching 
of the requested channel. Moreover, the spectrum bit map and the power value of link BC are also updated in its local database and a Resv message is sent to Node A. In turn, Node A executes the same tests over link $\mathrm{AB}$ after the receipt of the Resv message. Once verified, the hardware configuration is performed to its $A d d$ port in addition to channel power adaptation. Moreover, the spectrum bit map and the power value of link $\mathrm{AB}\left(P_{A B}(t)=P_{A B}(t)+\left(P_{\text {channel, } A B}^{\text {opt }} / C_{\text {adaptation, } A B C}\right)\right.$ are updated in its local database. Finally, the optical channel is established, and a connection setup confirmation is sent back to the network operator.

It important to note that every optical node sends its neighboring nodes a set of OSPF-TE LSAs messages. This regular update will naturally flood the changes over its outgoing links after the end of any signaling phase.

\section{SIMULATION SCENARIOS AND RESULTS}

\section{A. Simulation Setup and Scenarios}

In order to evaluate our proposed power control process, we developed a distributed GMPLS-based network simulator over OMNET++. It simulates OSPF-TE and RSVP-TE protocol messages and mechanisms, as explained in Section V. Moreover, it takes as input a network topology (links, spans, and amplifier types) and designs its optical links using our design method detailed in [17]. Finally, it fills in the OSPFTE database with the essential needed parameters $\left(P_{\text {channel, }}^{\text {opt }}\right.$, $P_{\text {design }, l}, P_{\text {margin }, l}, O S N R_{l}, P_{l}(t)$, etc). Simulations are performed over the 32 optical nodes and 42 optical links of the European backbone network shown in Fig. 8. Single mode fiber spans are assumed to be used (chromatic dispersion = $17 \mathrm{ps} . \mathrm{nm}^{-1} \cdot \mathrm{km}^{-1}$, fiber attenuation $=0.22 \mathrm{~dB} / \mathrm{km}$, nonlinearity coefficient $\left.=1 W^{-1} \cdot \mathrm{km}^{-1}\right)$.

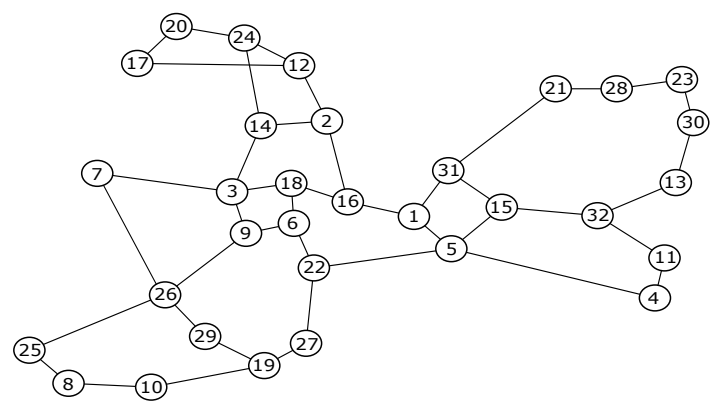

Fig. 8. European Backbone Network Topology.

Links are designed using the three amplifier types presented in Tab. I, assuming non identical span lengths that are randomly drawn according to a Gaussian distribution $\mathcal{N}(\mu=$ $100 \mathrm{~km}, \sigma=27 \mathrm{~km}$ ). Tab. I shows the amplifier portfolio used (several variable gain dual-stage amplifiers without mid-stage access), where $F_{1, n}$ and $F_{2, n}$ are the NF for the first and second stages, respectively, and $D_{n}$ denotes the power ratio for both stages to account for the difference between preamp and booster performance. Filtering penalties induced by transit across one optical node are $0.05 \mathrm{~dB}$ for the $50 \mathrm{GHz}$ (four slots of $12.5 \mathrm{GHz}$ ) channel spacing and $0.64 \mathrm{~dB}$ for the $37.5 \mathrm{GHz}$ (three slots of $12.5 \mathrm{GHz}$ ) [34].
TABLE I

AMPLIFIER MODELS

\begin{tabular}{|c|c|c|c|c|c|}
\hline Type & $P_{O A \_\max }(\mathrm{dBm})$ & $G_{O A \_\max }(\mathrm{dB})$ & $F_{1}(\mathrm{~dB})$ & $F_{2}(\mathrm{~dB})$ & Power ratio: $D(\mathrm{~dB})$ \\
\hline $\mathrm{A} 1$ & 17 & 30 & 5 & 6.5 & 3 \\
\hline $\mathrm{A} 2$ & 19 & 25 & 5.5 & 7 & 5 \\
\hline $\mathrm{A} 3$ & 20 & 23 & 6 & 7.5 & 7 \\
\hline
\end{tabular}

In order to simplify the results analysis, only $100 \mathrm{Gbit} / \mathrm{s}$ optical channels are established in all scenarios ( $T=100 \mathrm{Gbit} / \mathrm{s})$. The minimum accepted OSNR at the receiver side, using 0.1 $\mathrm{nm}$ noise reference bandwidth, including operational margins, is set to $15 \mathrm{~dB}$ for $100 \mathrm{Gbit} / \mathrm{s}$ QPSK modulation format with coherent detection and soft decision forward error correction (SDFEC), whatever the channel bandwidth (three or four slots of $12.5 \mathrm{GHz}$ ). Six scenarios are studied:

- Fixed-Grid (FG): This scenario represents today's core optical networks where no power information is communicated in the control plane. The power control is not activated in the path computation algorithm or in the protocol. The number of channels that can be set up on a given link is limited to 80 , where each channel occupies four contiguous slots $(4 * 12,5 \mathrm{GHz}=50 \mathrm{GHz})$.

- Fixed-Grid with power margins (FG4S_PV): In this scenario, the control plane is power aware and thus benefits from the extra power margin of every link $\left(P_{\text {margin }, l}\right)$ to set up channels in the limit of the $4.8 \mathrm{THz}$ bandwidth (C bandwidth). The power adaptation is set off, but the power verification is set on, and each channel occupies four contiguous slots.

- Fixed-Grid with power control and power margins (FG4S_PAPV): In this scenario, both power adaptation and power verification are allowed. Each individual channel power is tuned to the power satisfying the minimum acceptable OSNR value $\left(O S N R_{r e q}\right)$. Each channel occupies four contiguous slots.

- Flex-Grid (FX): This scenario is the same as FG but with channels occupying only three contiguous slots (filtering penalty is bigger than for FG scenario).

- Flex-Grid with power control and power margins (FX3S_PAPV): This scenario is the same as FG4S_PA+PV, but each channel occupies three contiguous slots.

- Flex-Grid with power control and power margins (FX34S_PAPV): This is the same as previous scenario, but with the possibility to choose three or four slots of 12.5 $\mathrm{GHz}$ for each $100 \mathrm{Gbit} / \mathrm{s}$ channel. The path computation algorithm first tries three slots of $12.5 \mathrm{GHz}$ for the channel setup. If the path is not physically feasible (probably because of the filtering penalty since it is higher for three-slot channels), the algorithm tries to establish the optical channel using four slots.

In this work, several Fixed-Grid and Flex-Grid scenarios are simulated. Therefore, in order to fairly compare them, we perform the same link design for eighty $100 \mathrm{Gbit} / \mathrm{s}$ QPSK channels over a $50 \mathrm{GHz}$ grid $(80 * 50 \mathrm{GHz}=4 \mathrm{THz}$ per link) for all scenarios. However, the full usable bandwidth of each link is set to $4.8 \mathrm{THz}$ (optical amplifiers usable bandwidth) as 
defined by the ITU-T.

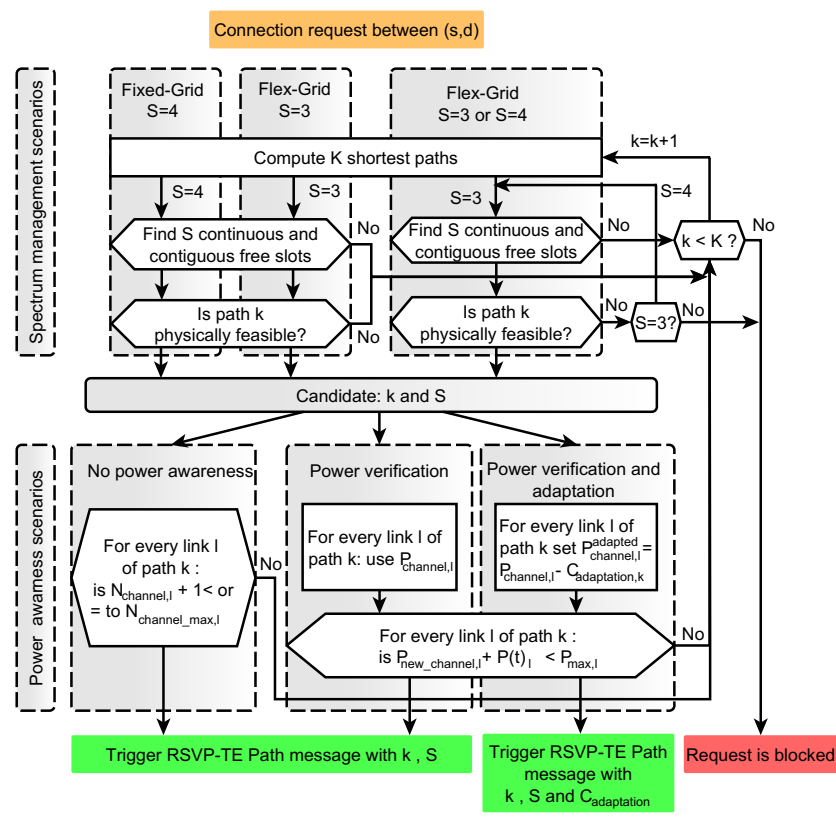

Fig. 9. Path computation algorithm of the simulated scenarios.

The path computation algorithm presented in IV is modified to enable the simulation of the different scenarios. We summarize in Fig. 9, the set of spectrum and power control tests applied during the execution of the path computation algorithm. Depending on the scenario, some tests are activated or deactivated. In this algorithm, the $K$ shortest paths can be computed for any request between any node pairs $(s, d)$. Therefore, when the $K$ paths have been computed, the ingress node executing the algorithm tries to establish the first path. If it is not possible, the second path is tested and so on. The connection request is blocked if no path from the $K$ computed paths can pass the set of tests. The $K$ paths are ordered in increasing length order. The algorithm selects the first path from $K$ that satisfies all the constraints (continuity, contiguity, physical feasibility, and if needed power feasibility). If one of the $K$ paths passes all the tests, the provisioning process is triggered with a set of channel parameters (path, slots, $\left.C_{\text {adaptation }}\right)$. The connection request is blocked if no path among the $K$ passes all the tests.

Note that in all scenarios, paths that exceed maximum reach (i.e., with OSNR below $O S N R_{r e q}$ ) are rejected and that our optical network does not implement regeneration (left for further study). Fifty simulation runs (each run with a different seed) are performed for each of the six scenarios. We simulate an incremental channel setup, where channels are established and never released (i.e., channel establishment until the network is fully loaded). It is important to note that, for every scenario, the same fifty seeds are used in order to simulate exactly the same sequence of optical connection requests. The results depicted in Fig. 10 and Fig. 11 are given by averaging the fifty simulation runs with a confidence interval of $95 \%$ (too small to be displayed on the figures). The connection request inter-arrival time at each node follows an exponential law with a value of 0.4 for its parameter. The source-destination pair of each request is randomly chosen among all network nodes according to a uniform distribution.

\section{B. Simulation Results}

We consider the cumulative blocking probability (CBR) a first evaluation criterion, which is the ratio of the total number of blocked requests over the total number of generated requests until a time $t$. Fig. 10 shows the CBR of the six scenarios as a function of the normalized spectrum occupation of the network, which is the ratio of the total occupied spectrum of all the links of the optical network until a time $t$ over the total spectrum of all the links. Note that, on each link, the spectrum occupation corresponds to the number of reserved slots of all channels, each one having three- or four-slot occupations depending on the scenario.

For all scenarios, the CBR at low occupation is not zero because of the rejected demands due to physical feasibility (paths longer than maximum reach). Not surprisingly, since FX and FX3S_PAPV have a larger filtering penalty, they block more demands at low occupation than the other scenarios. The CBR of the FX scenario increases rapidly with the spectrum occupation not only because of the physical feasibility blocking but also because of the limited number of channels over every link. When comparing FX and FG scenarios in terms of spectrum occupation, we notice that when the network is fully loaded (i.e., when no optical channel can be established), FX spectrum occupation represents $75 \%$ of that of FG. This result confirms the gain brought by Flex-Grid technology in terms of spectrum occupation. Moreover, FG and FG4S_PV have the same CBR until approximatively $65 \%$ of spectrum occupation. Over $65 \%$ occupation, the CBR of FG4S_PV is smaller because the network benefits from power awareness; it can accept more than 80 channels relying on the remaining power margins over the links.

Furthermore, FG4S_PAPV has a smaller CBR than FG and FG4S_PV because it can benefit not only from the power margin, but it can also create some reduction in power with our channel power adaptation process. The CBR of FG4S_PAPV stays below the CBR of FG and FG4S_PV starting from approximatively $26 \%$ of spectrum occupation. This means that even at low load, the power reduction enabled by our proposed power control mechanism can be useful.

Moreover, when investigating the optical power levels, we noticed that the FG4S_PAPV scenario is not limited by the optical power resource availability. In fact, the blocking was only due to physical feasibility and bandwidth availability, even at a high load. As explained earlier, the FX and FX3S_PAPV scenarios have bigger CBR at a low occupation ratio because they use only $37.5 \mathrm{GHz}$ spacing for establishing the 100 Gbit/s channels; the filtering penalty $(0.64 \mathrm{~dB})$ then reduces the number of feasible paths in the whole network. However, when network load increases, the FX3S_PAPV CBR is lower than the CBR of FG and FG4S_PV. This is explained first because, with three slots per channel, the network can accept more channels than with four slots. In addition, the optical power control process is able to sufficiently save power that is 


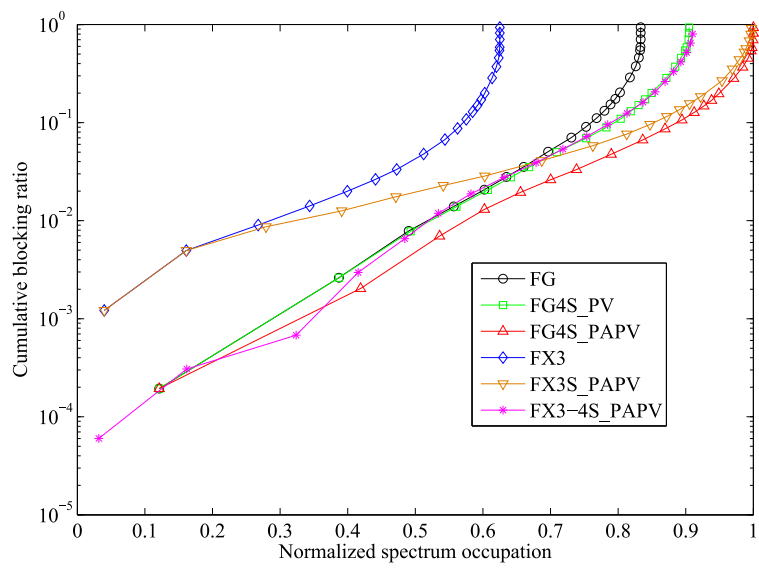

Fig. 10. Cumulative blocking ratio vs. normalized spectrum occupation.

required for these additional channels (despite the fact that the filtering penalty limits the performance and thus the amount of optical power reduction).

This analysis is confirmed with the FX3-4S_PAPV scenario. It has a CBR smaller than FX3S_PAPV for spectrum occupation lower than 0.65 . This is because paths that were rejected due to their non-physical feasibility with $37.5 \mathrm{GHz}$ are established here with $50 \mathrm{GHz}$. Nonetheless, this is paid with lower spectrum efficiency; the spectrum fragmentation caused by the mixing of $37.5 \mathrm{GHz}$ and $50 \mathrm{GHz}$ channels (no spectrum fragmentation awareness is used) prevents using the whole spectrum bandwidth, unlike FG4S_PAPV and FX3S_PAPV. This is also confirmed in Fig. 11.

It is important to note that the spectrum efficiency of the FX3S_PAPV is slightly smaller than FG4S_PAPV since some links still have spectrum resources, but their power resources are completely used at high loads. This is because setting up only three-slot channels not only increases the number of channels but also decreases the potential for power reduction over links. Power adaptation produces less power margins because of the higher filtering penalty $(0.64 \mathrm{~dB})$.

We notice that the amount of $P_{\text {margin,l }}$ over the links is too small to satisfy more than 80 channels with this network design (link power margins represent approximately $2.5 \%$ of the available power over the network). This means that when switching to Flex-Grid networks, the $P_{\text {margin,l }}$ will not be sufficient to handle the increase in the number of optical channels. In this situation, the power adaptation process is essential to save enough power to cancel the blocking for power reasons.

Moreover, we remarked that when the network is fully loaded (i.e. spectrally saturated), the remaining power over the entire network (sum of the remaining power over all network links) is high. We have $52 \%, 25 \%$, and $35 \%$ of remaining power for FG4S_PAPV, FX3S_PAPV, and FX3-4S_PAPV scenarios respectively. The value of the remaining power is high because all the $O S N R_{\text {margin,p }}$ of the established channels have been used to save optical power (i.e. reduce transmitted power). Therefore, for some channels, it is possible to use the existing $O S N R_{\text {margin }, p}$ for other purposes like using higher- order modulation format, to reduce the spectral occupation of the channel and thus increase links capacity. However, this increases the decision complexity in the control plane, since it should decide when to use the $O S N R_{\text {margin,p }}$ for power attenuation and when to use it to change the modulation format. This alternate decision policy is not addressed in this paper and it is left for future work.

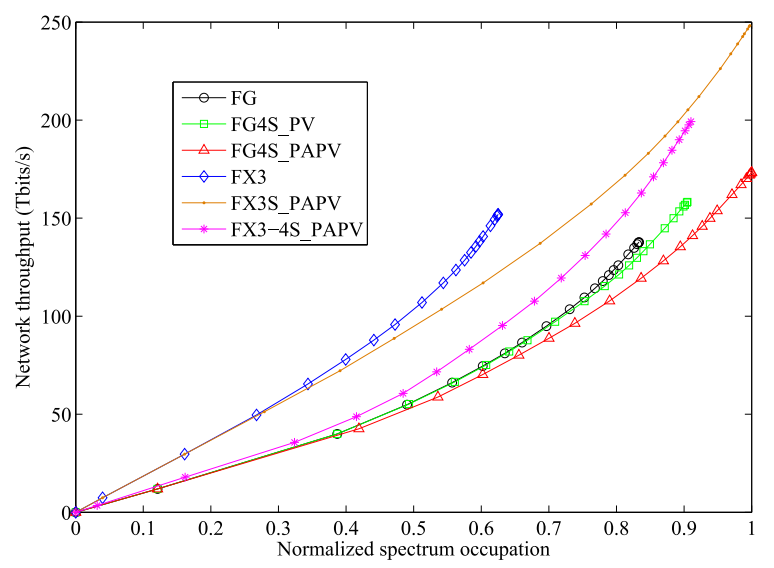

Fig. 11. Network throughput vs. normalized spectrum occupation.

Fig. 11 shows the network capacity (amount of $100 \mathrm{Gbit} / \mathrm{s}$ requests accepted and established) as a function of the normalized spectrum occupancy. Note that a four-slot $100 \mathrm{Gbit} / \mathrm{s}$ request going through three optical links (three-hop path), for example, will count as $100 \mathrm{Gbit} / \mathrm{s}$ on the y-axis and $3^{*} 4$ slots $(3 * 50 \mathrm{GHz})$ on the $\mathrm{x}$-axis. This explains why the FG4S_PV and FG4S_PAPV curves are below that of FG; both scenarios accept additional long path requests (i.e., paths with bigger hop numbers) at high load, because they can use more spectrum than FG (limited to 80 channels per link) thanks to the power control. This explanation also holds for the FX3S_PAPV scenario, which has much shorter paths on average than all the other scenarios (FX3S_PAPV curve is above that of FG).

The FX scenario carries approximately $152 \mathrm{Tbit} / \mathrm{s}$ of data traffic, which is more than the traffic carried with the FG scenario (137.8 Tbit/s). This result is expected since established connections in the FX scenario have shorter reaches and therefore occupy less bandwidth and slightly reduce the blocking due to the exceeded maximum channel number per link. The FG, FG4S_PV, and FG4S_PAPV reach at most 137.8 Tbit/s, $158.2 \mathrm{Tbit} / \mathrm{s}$, and $173.3 \mathrm{Tbit} / \mathrm{s}$, respectively, of carried traffic. Therefore, the power control has increased the capacity of the Fixed-Grid network by approximately $25 \%$.

As expected, the power control coupled with the use of the Flex-Grid in FX3S_PAPV greatly increases the network capacity to $248 \mathrm{Tbit} / \mathrm{s}$. This represents $80 \%$ of the capacity increase compared to FG (i.e., accounting for the $0.8 \mathrm{THz}$ more total spectrum resources compared to the $4 \mathrm{THz}$ of FG) and $45 \%$ when compared to FG4S_PAPV. We also note that the FX3-4S_PAPV scenario has a larger capacity than FG4S_PAPV, despite the fact that it can occupy less bandwidth because of spectrum fragmentation. 
All these results mean that channel power adaptation is an efficient mechanism to benefit from the link total spectrum bandwidth, without the need to redesign the existing optical network.

\section{Blocking Reasons}

To understand exactly what is happening during simulations, we plotted the reasons for request blocking for each scenario in bar charts and evaluated the effect of the number of shortest paths on the request blocking.

In our study, there are four blocking reasons:

- No available spectrum (No Spec): This type of blocking arises when no available continuous and contiguous slots are found over a path $p$.

- No sufficient OSNR (No OSNR): This type of blocking arises when the $O S N R_{e s t, p}$ of the calculated path is smaller than $O S N R_{r e q, p}$.

- No available power (No Pow): This type of blocking arises when no power resource is available in one link constituting the chosen optical path $p$.

- Maximum channel number exceeded (MXCE): This blocking reason is considered for FG and FX scenarios, where no power awareness exists in the control plane. Therefore, blocking arises when the channel number exceeds the maximum allowed (which is 80 here) over a link $l$ over the requested path $p$ (whatever the real remaining power or spectrum).

The blocking counting method is described as follows: for each connection request and its computed path $p$, if there are no available continuous and contiguous slots (over the path $p$ ), the blocking reason is counted as No Spec. However, if there are available slots but the $O S N R_{e s t, p}$ for the path $p$ is smaller than $O S N R_{r e q, p}$, the No OSNR blocking reason is counted. In the case where spectrum resources are available and the path is physically feasible $\left(O S N R_{e s t, p}>O S N R_{r e q, p}\right)$, but there is no power resource available in one of the links constituting the computed path $p$ (i.e., a link saturation may have occurred after adding the new optical channel), the No Pow blocking reason is counted. For FG and FX scenarios, since no power control is performed, the MXCE blocking reason is considered when the number of channels established over any link exceeds the maximum allowed. Therefore, No Spec is counted first, then No OSNR, and finally, MXCE.

To fairly compare the different scenarios, we recorded the results of simulation after 2000 connection requests were generated (same request sequence, same traffic, and same set of source and destination node pairs for all scenarios). Then, we plotted (in bar charts) the number of blocked requests per reason for blocking for each of the six scenarios. This is shown in Fig. 12 and Fig. 14 for Fixed-Grid and Flex-Grid scenarios, respectively, to ease visualization. In addition, these blocked requests per scenario are plotted as a function of the per channel hop number as shown in Fig. 13 and Fig. 15. Simulations are performed for one shortest path $(K=1)$ and for three shortest paths $(K=3)$.

1) One Shortest Path: Fig. 12 shows that, in the FG scenario, the MXCE reason is dominant. Indeed, no power information is available for the control plane, and the number of channels is the first blocking reason encountered when computing the path. Note that this does not mean that paths blocked due to the MXCE reason are otherwise feasible with respect to the continuity constraint or the physical feasibility constraint.

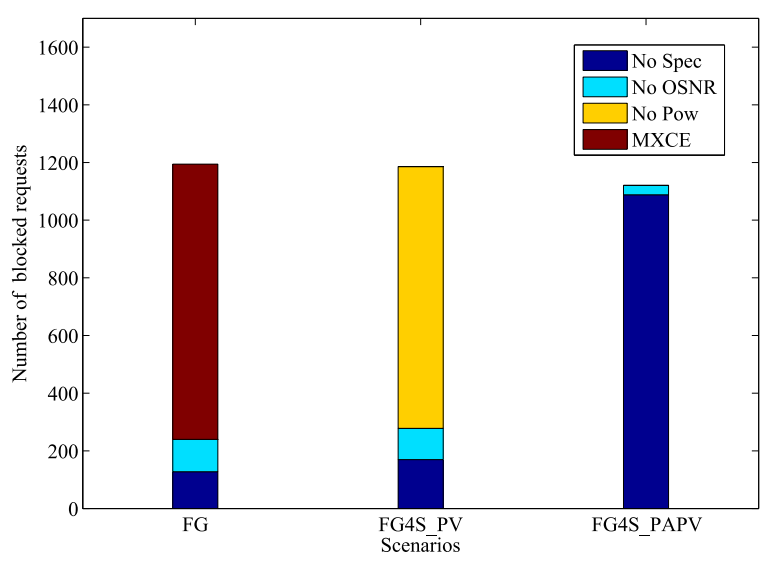

Fig. 12. Reasons for blocking in FG scenarios.

In the FG4S_PV scenario, since link power margin can be used, the amount of accepted request is increased, and the number of blocked requests is reduced (from 1194.4 to 1185.5 requests on average). Moreover, the No Pow reason is the main reason for blocking. This means that most of the requests have passed the continuity, the contiguity, and the physical feasibility tests but fail because the amount of power margin is not sufficient for a large number of them. This result confirm our first analysis that link power margin is not sufficient to avoid amplifiers saturation. However, some additional channels are accepted due to the use of power margins, leading to a bit more spectrum occupancy than in the FG (this explains the increase of the No spec blocking reason from 128 to 170 requests). This is also clear in Fig. 13, and it is independent from the hop number.

Fig. 12 shows that with the power control process in FG4S_PAPV, the blocking occurs for two reasons: No OSNR and No Spec. This is explained by the fact that the power control process is capable of reducing link power; therefore, link power saturation is no longer occurring. Power adaptation frees more optical power resources than required by the requests. Power is no longer a limitation in this case. As a result, more connections are accepted, and less are blocked as it can be seen in Fig. 13. Therefore, network links are more spectrally occupied.

This explains why the number of No OSNR blocking in the FG4S_PAPV scenario is smaller than the No OSNR blocking number in the FG and FG4S_PV scenarios: more requests are counted as blocked due to spectrum resources first, even if these requests do not pass the physical feasibility test. This somehow masks part of the No OSNR blocking reason in the FG4S_PV scenario (because of the blocking counting method).

Fig. 14 shows that in the FX scenario, there is no blocking due to No Spec, since the use of three slots for each 100 


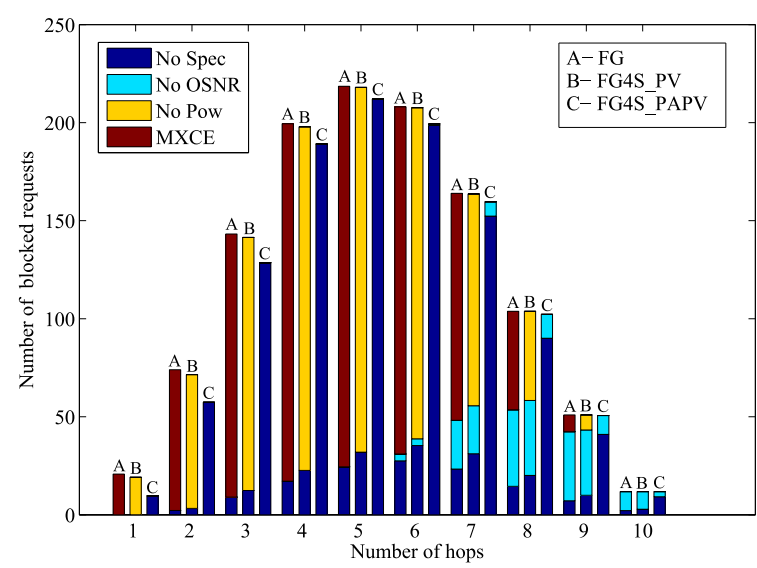

Fig. 13. Reasons for blocking per hop number in FG scenarios.

Gbit/s channel has reduced the used spectrum in the network; therefore, there is sufficient available spectrum for all requests. This confirm the spectral gain promises when using the FlexGrid technology. However, the blocking reasons are instead due to No OSNR since the filtering penalty is bigger than that of the FGs scenarios. Of course, as for the FG scenario, there are always some blockings due to MXCE, since power information is not available to the control plane.

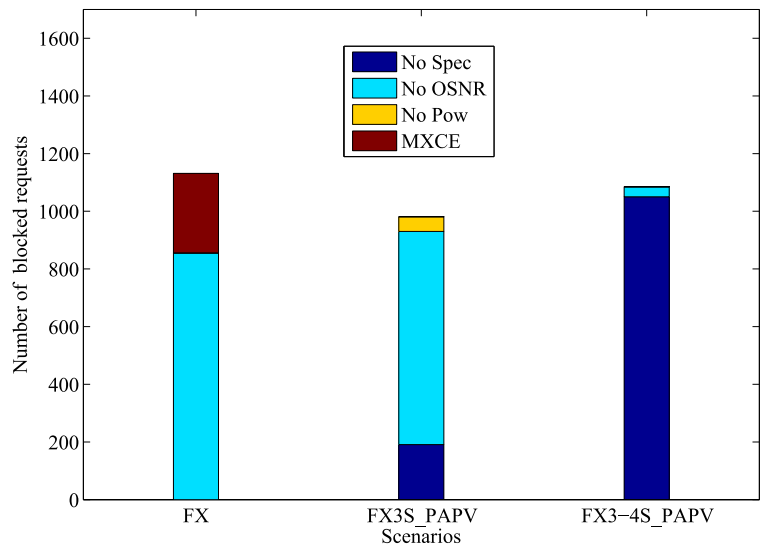

Fig. 14. Reasons for blocking in FX scenarios.

We notice that in the FX_PAPV scenario, the dominant blocking reason is also No OSNR because of the high filtering penalty but with a smaller number (739.2 requests) in comparison with that of FX (855.7 requests). The reason behind that is the same as explained in the previous paragraph when comparing the number of No OSNR blockings in the FG and FG4S_PAPV scenarios. In this scenario (FX3S_PAPV), the activation of the power control process increases the number of established channels, as shown in Fig. 14 and Fig. 15; therefore, some optical links are fully occupied (up to 128 channels rather than 80 in the FX scenario). This explains the appearance of No Spec blockings.

At the same time, we can see in Fig. 14 that No Pow blocking arises in the FX3S_PAPV scenario. Moreover, this blocking reason is limited to requests with a small number of hops as shown in Fig. 15. In fact, the high filtering penalty reduces channel performance and the quantity of power that can be saved through the power control process, since less $O S N R_{\text {margin,p }}$ is saved per channel; and more power resources are consumed. Thus, the amount of freed optical power resources is not enough to cope with the available spectrum resources and requests. We can notice also that the requests with long paths are more likely to be blocked due to the OSNR limit, and their blocking reason is considered No OSNR, even if there is a lack of power resources (due to the counting method).

It is noteworthy that, since the traffic is uniformly distributed among all fibers, this lack of power resources (and the lack of spectral resources) will appears especially on links like the one between Node 1 and Node 16, and between Node 5 and Node 22 of the Fig. 8. This is because these links interconnect two parts of the network. Therefore, a special attention should be dedicated to these links, which is one of our future works.

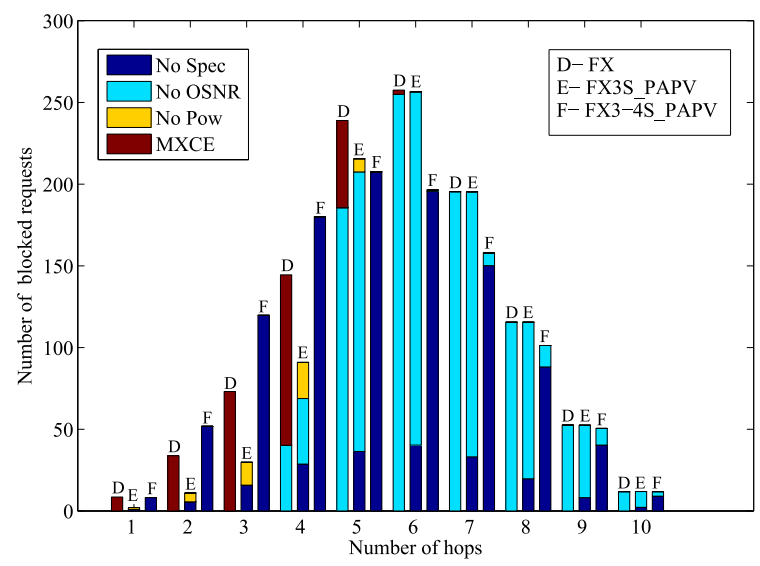

Fig. 15. Reasons for blocking per hop number in FX scenarios.

Lastly, the FX3-4S_PAPV scenario suffers from spectrum limitation. This is because of spectrum fragmentation since a mix of three- and four-slot channels are used; thus, network links cannot be fully occupied. In addition, four slots channels occupy more spectrum and have bigger number of hops. Therefore No Spec blocking is dominant and the No Pow blocking reason never arises. This is in line with the effort made in the literature to reduce spectrum fragmentation.

Fig. 15 shows that the FX and FX3S_PAPV scenarios accept more requests with a small number of hops (less blocking for paths with a small number of hops) because of the freed spectrum. Instead, in FX3-4S_PAPV, a higher number of blockings appears for requests with hop counts lower than five because more requests with a large number of hops are accepted. Moreover, in FX3-4S_PAPV, the number of No OSNR blocking is reduced. This is explained by the fact that connection requests that are not physically feasible with three slots (because of the high filtering penalty) are established with four slots.

We can deduce from these results that the strategies used for channel establishment (i.e. selection of the transponder type, 
channel power, modulation format, spectral occupation and baud-rate) is very important in order to exploit the capacity of network links. Therefore, more intelligent routing algorithm is needed in order to benefits from the Flex-Grid technology gain promises.

2) Three Shortest Paths: To complete the evaluation of our power control process, the path computation algorithm is improved by introducing path diversity (i.e., $K$ shortest paths computation) and simulations are repeated with $K=3$ shortest paths.

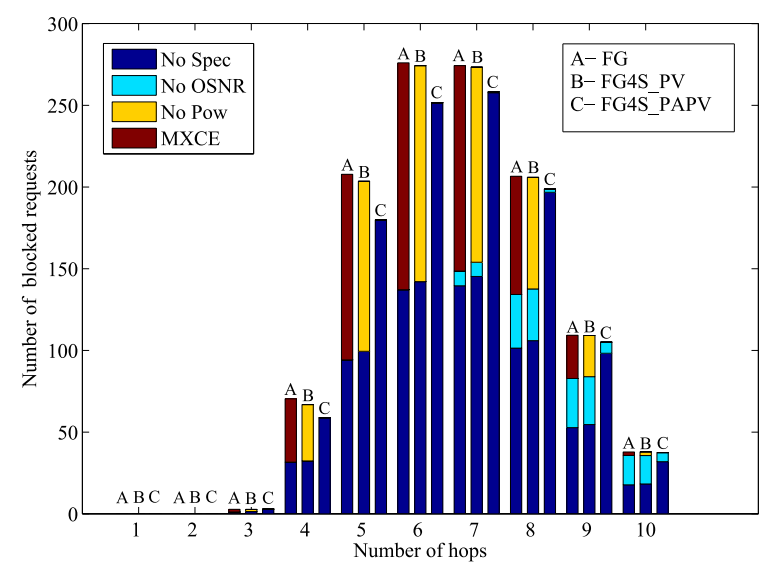

Fig. 16. Reasons for blocking per hop number in $\mathrm{FG}$ scenarios with $\mathrm{K}=3$.

Fig. 16 and Fig. 17 plot in bar charts the number of blocked requests in function of the per channel hop number. In these simulations, the blocking reason is recorded for the last tested path. This is why no blocking is recorded for one- and two-hop paths as shown in Fig. 16 and Fig. 17. This is expected because the established connections have longer reaches on average with $K=3$. Accordingly, connection requests occupy more spectrum and therefore network throughput for all scenarios is reduced and the No Spec blocking number increases compared with $K=1$.

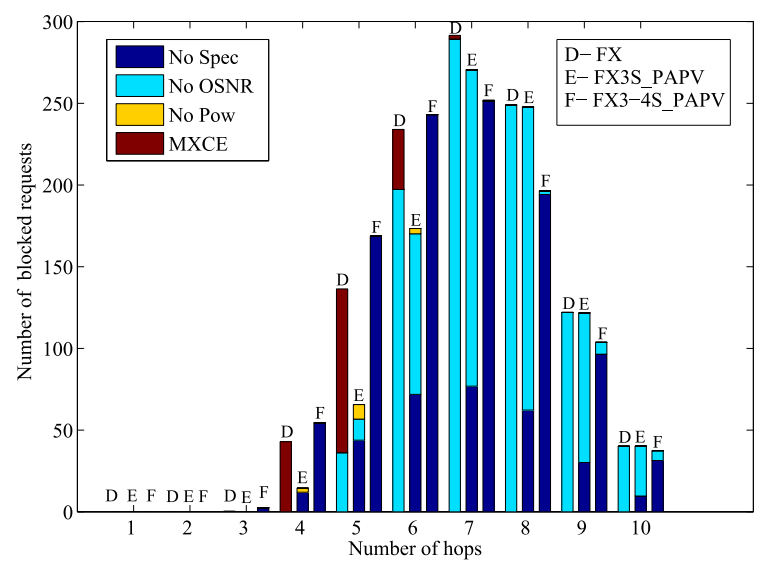

Fig. 17. Reasons for blocking per hop number in $\mathrm{FX}$ scenarios with $\mathrm{K}=3$.

We note that the CBR values when $K=3$ are slightly reduced for all scenarios in comparison with the shortest path
$K=1$. However, we obtain approximately the same behavior and shape for $K=1$ and $K=3$ (figures are not included due to lack of space).

It is interesting to increase the number of calculated shortest paths in order to avoid blocking in case there is a lack of spectrum and power resources over optical links. Indeed, this reduces the network blocking, but, at the same time, more resources are consumed on average. However, Fig. 17 shows that the No Pow blocking reason in FX3S_PAPV is not avoided even for just 2000 generated requests.

This result shows that, even with a routing algorithm, which takes advantage of path diversity, No Pow blocking could not be avoided. Because as explained before, the links interconnecting different parts of the network will always be problematic. This is why, it is important to include power information in the control plane to efficiently manage network resources and therefore define strategies to avoid this kind of blocking.

\section{CONCLUSION}

In this paper, we address the optical amplifier power limitation issue that an optical network operator will face when migrating networks from Fixed-Grid to Flex-Grid networks. We recall our link design method that allows specifying the power information of optical layers that are essential for the control plane. A channel power control process is proposed in addition to a path computation algorithm that integrates power verification and power adaptation tests. We show how the whole power control process can be implemented into a distributed GMPLS-based control plane and propose new extensions for OSPF-TE and RSVP-TE protocols to include power information and to integrate power awareness. Simulation results reveal that the power control process is an efficient way to benefit from Flex-Grid capacity promises while maintaining the use of legacy amplifiers without the need to redesign any link in the network. In addition, it helps to efficiently manage link power resources and to avoid power saturation, which is certainly unacceptable during network operation.

It is important to emphasize that our power control process is completely independent from link design, OSNR estimator, or control plane protocol. Any other link design method associated with any OSNR estimator could be used to perform the power control. In addition, this process could be used for an already deployed network, where established channels are adjusted to fit operator requirements while monitoring their error rates. At the same time, it could also be considered for new Flex-Grid networks under construction, where we anticipate the deployment of power controlled channels to liberate margins and thus increase network throughput.

Future work will include a performance evaluation using other network topology, in addition to optical regeneration. The power control process will also be evaluated in the dynamic case where optical connections are established and released, including different modulation formats/rates with different optical powers. 


\section{ACKNOWLEDGMENTS}

This work was partly supported by the DGCIS, in the frame of the CELTIC-Plus project SASER-SIEGFRIED.

\section{REFERENCES}

[1] O. Gerstel, M. Jinno, A. Lord, and S. B. Yoo, "Elastic optical networking: A new dawn for the optical layer?" Communications Magazine, IEEE, vol. 50, no. 2, pp. s12-s20, 2012.

[2] P. Wright, A. Lord, and L. Velasco, "The network capacity benefits of Flexgrid," in Optical Network Design and Modeling (ONDM), 2013 17th International Conference on. IEEE, 2013, pp. 7-12.

[3] "Spectral grids for WDM applications: DWDM frequency grid." ITU-T Recommendation G.694.1, 2012.

[4] D. Amar, N. Brochier, E. L. Rouzic, J. L. Auge, C. Lepers, B. Cousin, and M. Kanj, "Link design and legacy amplifier limitation in flex-grid optical networks," IEEE Photonics Journal, vol. 8, no. 2, pp. 1-10, April 2016.

[5] P. Poggiolini, "The GN Model of Non-Linear Propagation in Uncompensated Coherent Optical Systems," Journal of Lightwave Technology, vol. 30, no. 24, pp. 3857-3879, Dec. 2012.

[6] F. Vacondio, C. Simonneau, L. Lorcy, J.-C. Antona, A. Bononi, and S. Bigo, "Experimental characterization of Gaussian-distributed nonlinear distortions," in European Conference on Optical Communications. Optical Society of America, 2011, pp. We-7.

[7] A. Carena, V. Curri, G. Bosco, P. Poggiolini, and F. Forghieri, "Modeling of the Impact of Nonlinear Propagation Effects in Uncompensated Optical Coherent Transmission Links," Journal of Lightwave Technology, vol. 30, no. 10, pp. 1524-1539, May 2012.

[8] G. Bosco, A. Carena, R. Cigliutti, V. Curri, P. Poggiolini, and F. Forghieri, "Performance prediction for WDM PM-QPSK transmission over uncompensated links," in Optical Fiber Communication Conference. Optical Society of America, 2011.

[9] D. J. Ives and S. J. Savory, "Transmitter optimized optical networks," in National Fiber Optic Engineers Conference. Optical Society of America, 2013, pp. JW2A-64.

[10] D. J. Ives, P. Bayvel, and S. J. Savory, "Adapting Transmitter Power and Modulation Format to Improve Optical Network Performance Utilizing the Gaussian Noise Model of Nonlinear Impairments," Journal of Lightwave Technology, vol. 32, no. 21, pp. 4087-4096, Nov 2014.

[11] A. Mitra, A. Lord, S. Kar, and P. Wright, "Effect of link margins and frequency granularity on the performance and modulation format sweet spot of multiple flexgrid optical networks," in Optical Fiber Communications Conference and Exhibition (OFC), 2014, March 2014, pp. 1-3.

[12] D. J. Ives, P. Bayvel, and S. J. Savory, "Assessment of options for utilizing snr margin to increase network data throughput," in Optical Fiber Communications Conference and Exhibition (OFC), 2015, March 2015, pp. 1-3.

[13] A. Nag, M. Tornatore, and B. Mukherjee, "Power management in mixed line rate optical networks," in Integrated Photonics Research, Silicon and Nanophotonics and Photonics in Switching. Optical Society of America, 2010.

[14] A. Bononi, P. Serena, and A. Morea, "Regeneration savings in coherent optical networks with a new load-dependent reach maximization," in 2014 The European Conference on Optical Communication (ECOC), Sept 2014, pp. 1-3.

[15] R. Martinez, R. Casellas, R. Munoz, and T. Tsuritani, "Experimental translucent-oriented routing for dynamic lightpath provisioning in gmpls-enabled wavelength switched optical networks," Journal of Lightwave Technology, vol. 28, no. 8, pp. 1241-1255, April 2010.

[16] R. Casellas, R. Muñoz, J. M. Fàbrega, M. S. Moreolo, R. Martínez, L. Liu, T. Tsuritani, and I. Morita, "Gmpls/pce control of flexigrid dwdm optical networks using co-ofdm transmission [invited]," IEEE/OSA Journal of Optical Communications and Networking, vol. 4, no. 11, pp. B1-B10, Nov 2012.

[17] M. Kanj, E. Le Rouzic, D. Amar, J.-L. Auge, B. Cousin, and N. Brochier, "Optical power control to efficiently handle flex-grid spectrum gain over existing fixed-grid network infrastructures," in International Conference on Computing, Networking and Communications (Accepted), 2016.

[18] D. Amar, M. Kanj, J.-L. Auge, N. Brochier, E. Le Rouzic, C. Lepers, and B. Cousin, "On legacy amplifier limitation in flexgrid optical networks," in International Conference on Photonics in Switching, 2015.
[19] P. Poggiolini, G. Bosco, A. Carena, R. Cigliutti, V. Curri, F. Forghieri, R. Pastorelli, and S. Piciaccia, "The LOGON strategy for lowcomplexity control plane implementation in new-generation flexible networks," in Optical Fiber Communication Conference. Optical Society of America, 2013, pp. OW1H-3.

[20] J. Auge, "Can we use flexible transponders to reduce margins?" in $O p$ tical Fiber Communication Conference/National Fiber Optic Engineers Conference, 2013.

[21] A. Mitra, S. Kar, and A. Lord, "Effect of frequency granularity and Link Margin at $100 \mathrm{~g}$ and beyond Flexgrid Optical Networks," in Communications (NCC), 2014 Twentieth National Conference on. IEEE, 2014, pp. 1-5.

[22] A. Mitra, A. Lord, S. Kar, and P. Wright, "Effect of link margin and frequency granularity on the performance of a flexgrid optical network," Optics express, vol. 22, no. 1, pp. 41-46, 2014.

[23] F. Vacondio, O. Rival, C. Simonneau, E. Grellier, A. Bononi, L. Lorcy, J.-C. Antona, and S. Bigo, "On nonlinear distortions of highly dispersive optical coherent systems," Optics Express, vol. 20, no. 2, pp. 1022-1032, 2012.

[24] E. Mannie, "Generalized multi-protocol label switching (gmpls) architecture," IETF RFC 3945, October 2004.

[25] X. Zhang, R. Casellas, O. G. de Dios, and D. Ceccarelli, "Gmpls ospf-te extensions in support of flexi-grid dwdm networks," Working Draft, IETF Secretariat, Internet-Draft draft-ietf-ccamp-flexible-grid-ospf-ext04, April 2016. [Online]. Available: http://www.ietf.org/internetdrafts/draft-ietf-ccamp-flexible-grid-ospf-ext-04.txt

[26] F. Zhang, X. Zhang, A. Farrel, O. G. de Dios, and D. Ceccarelli, "Rsvpte signaling extensions in support of flexi-grid dense wavelength division multiplexing (dwdm) networks," IETF RFC 7792, March 2016.

[27] G. Martinelli, X. Zhang, G. Galimberti, A. Zanardi, D. Siracusa, F. Pederzolli, Y. Lee, and F. Zhang, "Information model for wavelength switched optical networks (wsons) with impairments validation," IETF Internet-Draft, draft-ietf-ccamp-wson-iv-info-02, October 2015, work in progress. [Online]. Available: http://www.ietf.org/internet-drafts/draftietf-ccamp-wson-iv-info-02.txt

[28] "Physical transfer functions of optical network elements." ITU-T Recommendation G.680, 2007.

[29] Y. Lee, G. Bernstein, D. Li, and G. Martinelli, "A framework for the control of wavelength switched optical networks (wsons) with impairments," IETF RFC 6566, March 2012.

[30] I. Hussain, R. Rao, M. Sosa, and A. Dhillon, "Te extensions to ospf for gmpls control of flex-grid networks," Working Draft, IETF Internet-Draft draft-dhillon-ccamp-flexgrid-ospfte-ext-00, March 2014. [Online]. Available: http://www.ietf.org/internet-drafts/draftdhillon-ccamp-flexgrid-ospfte-ext-00.txt

[31] F. Zhang, Y. Lee, J. Han, G. Bernstein, and Y. Xu, "Ospf-te extensions for general network element constraints," IETF RFC 7580, June 2015.

[32] G. Martinelli and A. Zanardi, "Gmpls signaling extensions for optical impairment aware lightpath setup," Work in progress, IETF InternetDraft draft-martinelli-ccamp-optical-imp-signaling-03, October 2010. [Online]. Available: http://www.ietf.org/internet-drafts/draft-martinelliccamp-optical-imp-signaling-03.txt

[33] B. Braden, L. Zhang, S. Berson, S. Herzog, and S. Jamin, "Resource reservation protocol (rsvp) - version 1 functional specification," IETF RFC 2205, September 1997. [Online]. Available: http://www.rfceditor.org/rfc/rfc2205.txt

[34] D. Amar, E. Le Rouzic, N. Brochier, J.-L. Auge, C. Lepers, N. Perrot, and S. Fazel, "How problematic is Spectrum Fragmentation in operator's Gridless network?" in International Conference on Optical Network Design and Modeling, 2014. IEEE, 2014, pp. 67-72.

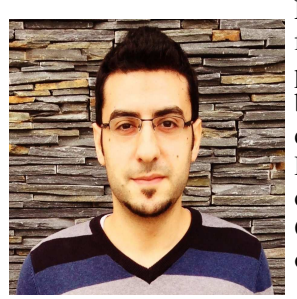

Mohamad Kanj received an engineering degree from Superior National School of Computer and Applied Mathematics of Grenoble (ENSIMAG), Grenoble, France, in 2012. He is currently a research engineer at $\mathrm{b}<>$ com and a Ph.D. candidate in Rennes 1 University. His research interests concern computer and optical network architecture, FlexGrid technology, and GMPLS protocol suite for optical networking. 


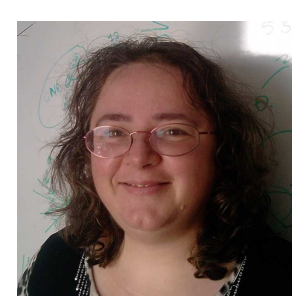

Esther Le Rouzic Esther Le Rouzic received a telecommunication degree from Telecom Bretagne, Bretagne, France, an M.Sc. degree from the University College London, London, U.K., in 1996, and a Ph.D. degree in electronics and communications from Telecom Paris, Paris, France, in 1999. She joined Orange Labs (previously France Telecom R\&D), Lannion, France, in 2000, where she has been working on optical networks. Her research interests include sub-wavelength photonic switching, flexible optical networks, optical networks automation, and energy-efficient solutions for core and metro networks.

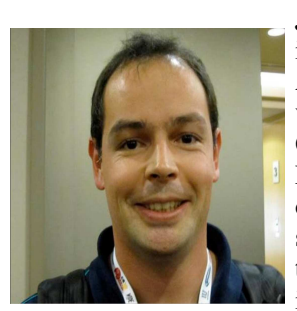

Julien Meuric graduated from the French engineering school "Ecole Nationale Supérieure des Sciences Appliquées et de Technologie" (ENSSAT). After various activities on packet networks, he joined Orange Labs (previously France Telecom R\&D), Lannion, France, in 2004, where he has worked on metropolitan and optical core networks. His research interests include control plane integration in transmission networks (such as SDH or WDM) and interactions with packet layers. As standardization senior manager, he contributes to coordinating the standardization of transport network technologies and is personally involved in standardization around GMPLS control protocols in corresponding IETF working groups (CCAMP, L1VPN, MPLS...). Besides technical contributions, he also acts in IETF as co-chairman of the Path Computation Element (PCE) working group.

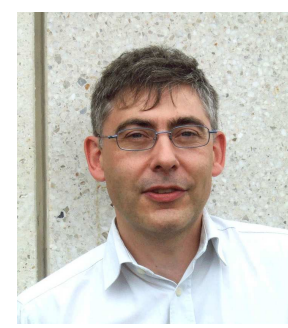

Bernard Cousin has been, is a Professor of Computer Science at the University of Rennes 1, in France since 1992. Bernard Cousin received, in 1987, his Ph.D. degree in computer science from the University of Paris 6. Between 1985 and 1992, he has successively been Assistant Professor at University of Paris 6 and Associate Professor at University of Bordeaux 1. Currently, he is at the head of a research group on Advanced Networking. He is a member of IRISA (a CNRS-University joint research laboratory located at Rennes in France). He has coauthored $150+$ papers published in international journals and conferences. His research interests include next generation Internet, green networking, all-optical networks, wireless networks, dependable networking, high speed networks, traffic engineering, multicast routing, network QoS management, network security and multimedia distributed applications.

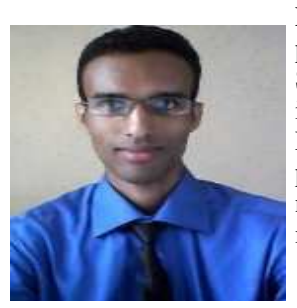

Djamel Amar received his master's degree in computer sciences and telecommunications from Paul Sabatier University, Toulouse, France, in 2012. He is currently a research engineer at Orange Labs, Lannion and a Ph.D. candidate with Telecom Sudparis. His main research interests concern transport network architectures, network performance assessment, and flex-grid technology. 